\title{
Validity, utility and acceptability of borderline personality disorder diagnosis in childhood and adolescence: survey of psychiatrists
}

\author{
Mark Griffiths ${ }^{1}$
}

The Psychiatrist (2011), 35, 19-22, doi: 10.1192/pb.bp.109.028779

${ }^{1}$ Child and Family Psychological Health Service, Aneurin Bevan Health Board, Newport, UK

Correspondence to Mark Griffiths (mark.griffiths2@wales.nhs.uk)
Aims and method To establish the views of child and adolescent psychiatrists $(n=52)$ regarding the conceptual and empirical validity, clinical utility and acceptability of the diagnosis of borderline personality disorder in child and adolescent populations. A questionnaire survey was carried out.

Results The child and adolescent psychiatrists' perception of the validity of borderline personality disorder for adult populations was relatively high ( $82 \%$ felt it to be valid). Significantly fewer of those considered borderline personality disorder to be valid for adolescent populations (37\%). Strikingly different results were obtained when the questions related to child ( $<12$ years) populations $(2 \%)$.

Clinical implications Given the views expressed by these consultant child and adolescent psychiatrists, it would seem appropriate to approach with caution suggestions that the borderline personality disorder category should have extended use with adolescent and child populations.

Declaration of interest None.
Borderline personality disorder is often considered a controversial diagnostic category, on a number of grounds, when applied to adult populations. ${ }^{1-4}$ In spite of this, emerging literature claims that the category is valid for use in adolescent, and even child, populations. ${ }^{4-6}$

The label 'borderline personality disorder' discussed in this article refers to the category that has been operationalised in DSM-IV. ${ }^{7}$ Many of the points made are applicable to the comparable category of 'emotionally unstable personality disorder', which is included in ICD-10. ${ }^{8}$ DSM-IV allows borderline personality disorder to be diagnosed in adolescents when maladaptive traits have been present for at least 1 year, are persistent and all-encompassing, and are not likely to be limited to a developmental stage or an episode of an Axis I disorder. However, ICD-10 does not recommend diagnosis for teenagers under 17 years of age: 'It is therefore likely that the diagnosis of personality disorder will not be appropriate before the age of 16 or 17 years'.

Validity judgements of psychiatric classification systems in general, and of specific diagnostic categories in particular, have come to rely almost solely on empirical considerations. ${ }^{9,10}$ Over the past decade or so a counterbalancing body of literature has presented arguments for the foregrounding and scrutiny of a range of conceptual considerations in addition to the more familiar and established empirical approaches to the issues of validity. ${ }^{11}$ Such considerations require an analysis of the underlying assumptions and value judgements involved in the content and processes of classification and the determination of disorder status in psychiatry. ${ }^{12-14}$

The distinction between conceptual and empirical validity, although often neglected in contemporary accounts, was acknowledged by the psychiatric classification scholar Robert Kendall. Kendall \& Jablensky ${ }^{15}$ considered it a rarity for psychiatric disorders to clearly demonstrate sufficient conceptual and empirical validity to be definitively accorded the legitimacy of 'true' disorders. However, they asserted that the majority of diagnostic categories should be retained on pragmatic grounds, as they provided practitioners with clinical utility.

Critiques of the borderline personality disorder construct in adults and younger populations have focused on conceptual issues, ${ }^{2}$ empirical evidence ${ }^{16-21}$ and clinical utility perspectives. ${ }^{22}$ In addition, an increasing body of user-led qualitative research, alongside a greater emphasis within the literature on value-based approaches to mental health provision, has highlighted the need for clinicians to consider the views of various different stakeholders and ensure that practice is experienced by service users as acceptable. ${ }^{3,23}$ The label 'borderline personality disorder' has been described almost invariably in negative terms (including 'stigmatising', 'pejorative', 'marginalising' and 'objectifying') by many of those people who have attracted the diagnosis and have been asked about their experiences. ${ }^{24}$

Although the views of professionals involved in the assessment and treatment of individuals who may receive the diagnosis of borderline personality disorder have been 
studied less, the perceptions of nurses and psychiatrists towards individuals with such a diagnosis have been found to be negative and stereotypical., ${ }^{3,25}$

This study aimed to establish the views of child and adolescent psychiatrists who would be the main gatekeepers of the borderline personality disorder diagnosis if the mainstream view, as enshrined in practice guidelines such as those of the National Institute for Health and Clinical Excellence (NICE), moves in the direction of suggesting that the category is valid, useful and acceptable for child and adolescent populations. The survey was designed to elicit views about the conceptual and empirical validity, clinical utility and acceptability of the diagnosis.

\section{Method}

The questionnaire involved respondents answering 17 questions. Twelve of the questions involved a Likert scale, two were yes/no response questions, one was a closed question, and two were open questions with space left available to add comments.

Delegates who attended the annual general meeting at the 2009 residential child psychiatry conference, all of whom were child and adolescent psychiatrists, were asked to complete the questionnaire. Of 85 delegates, 52 returned the questionnaire (61\%). Comments to the open questions were analysed and grouped according to emerging themes.

\section{Results}

Of the respondents, $82 \%$ accepted the overall validity of borderline personality disorder for adult populations. The subquestions that separated conceptual (87\% agreed) and empirical (67\% agreed) considerations of validity showed similar profiles.

Significantly fewer respondents considered borderline personality disorder to be valid for adolescent populations (37\%). The subquestions showed similar profiles, with empirical validity being considered less than conceptual validity ( $40 \%$ v. $29 \%)$.

Strikingly different results were obtained when the questions related to child ( $<12$ years) populations: only $2 \%$ agreed it was a valid category. The respondents considered the diagnosis neither conceptually nor empirically valid for this age group.

Specific questions were asked about the criteria used in the diagnosis: $62 \%$ considered that borderline personality disorder included pejorative language, $63 \%$ believed the category involved value judgements, and $80 \%$ viewed it as stigmatising. Forty-six per cent of respondents thought that a diagnosis of borderline personality disorder was necessary to provide appropriate interventions, but only $14 \%$ believed the diagnosis to be as valid as other categories used for child and adolescent populations. Most of the sample considered that it would be more appropriate to use a dimensional rather than a categorical classification system for personality difficulties.

Only $23 \%$ of respondents use the diagnosis in regular clinical practice. Of those that do, $60 \%$ feed back their diagnostic formulation to young people and their families.

There was no significant relationship between the answers provided by respondents and the tier of child and adolescent mental health services (CAMHS) within which they work.

The option of providing written comments was taken up by 39 of the 52 respondents. Those who utilised the diagnosis and viewed it positively spoke of the following themes:

'The diagnosis can help families and young people understand their experiences and difficulties.'

'It may also help young people access appropriate interventions such as dialectical behaviour therapy (DBT).'

A group of psychiatrists who considered the diagnosis more neutrally made numerous suggestions that their formulations may occasionally provide a clear diagnosis but more commonly involve descriptions of 'emerging personality difficulties', which were perceived to be less stigmatising and suggesting more fluidity/modifiability of clinical course. Some recognised that they encountered adolescents with characteristic problems associated with the diagnostic criteria but were nonetheless sceptical of the utility of applying the diagnosis in routine clinical practice: 'The existence of the profile of difficulties is less dubious than the conceptualising of it as a valid diagnostic category.'

The majority of comments involved negative views of the use of borderline personality disorder with child and adolescent populations. The themes described included the following:

- the label may have stigmatising, marginalising and objectifying effects on young people

- making the diagnosis can lead to a worsening of the difficulties

- the diagnosis leads to therapeutic pessimism and a belief that change is impossible.

The diagnosis is conceptually problematic, as it omits crucial developmental factors and makes assumptions about the enduring nature of certain personality variables, which is considered developmentally naive in adolescent populations. Furthermore, the criteria allow for too much heterogeneity in the profiles of difficulties presented by those individuals who may attract the diagnosis, thereby reducing its clinical utility.

Many of those unconvinced that a diagnosis of borderline personality disorder adds anything to their clinical practice provided many alternative suggestions for the formulation of young people with difficulties that might be conceptualised as borderline personality disorder. They frequently stated that they did not require a diagnostic framework to identify an individual's profile of difficulties or to devise therapeutic plans for the individual. Complex descriptive developmental formulations, including factors such as trauma, attachment difficulties, identity confusions, strengths and weaknesses, relational problems and emotional regulation difficulties, were viewed as more appropriate for this group of young people rather than a diagnosis that was considered by some to be a 'trite', 'abhorrent', 'insulting', 'stigmatising' or 'simplifying' label.

\section{Discussion}

The profiles of responses to the questions and the additional comments suggest that consultant child and adolescent psychiatrists in the UK view the category of borderline personality disorder as conceptually problematic, empirically insufficiently supported, lacking in clinical utility, and tending 
towards being perceived as unacceptable for individuals and their families. These views are held considerably more strongly when adolescent populations are considered. The results suggest that child psychiatrists do not consensually accept that the borderline personality disorder diagnostic construct is valid for an adolescent population. They view it almost unanimously as an inappropriate and invalid category for use with children under the age of 12 years.

Interestingly, even those clinicians who consider the category to be valid do not all choose to feed back their diagnostic formulations to young people (60\% do provide such feedback). This restrained transparency seems to reflect clinicians' awareness of the perceived unacceptability of a label that the majority of respondents considered as stigmatising, value-laden and pejorative.

A large body of literature attests to the rich array of explanatory models and therapeutic approaches that can be drawn upon to help individuals who present with profiles of difficulty that meet diagnostic criteria for borderline personality disorder. These include psychoanalytic psychotherapy (especially transference-focused and mentalisationbased approaches), modified cognitive-behavioural therapy (especially schema-focused), attachment-based approaches, cognitive analytic therapy, dialectical behaviour therapy, therapeutic communities, solution-focused therapy, narrative therapy and the cautious use of psychotropic medications (for a useful summary, see Bateman \& Fonagy ${ }^{26}$ ). Each approach advocates the use of detailed formulations (each model differing on the details, preferred emphases and theoretical concepts employed in these formulations), with individualised profiles emerging leading to a therapeutic management plan. None of the approaches requires the formulation to be in categorical diagnostic form in order to be used successfully. In addition, all of these approaches stress the vital importance of engaging well with these hard-to-reach individuals who, almost universally, struggle to trust adults in perceived authority positions. To achieve this goal, practitioners need to focus a great deal of attention on facilitating the development of therapeutic relationships and considering carefully how to communicate the explanatory and therapeutic formulations that will be used to guide management plans.

The above conclusions and priorities derived from the therapy literature are supported by a large, and increasing, amount of literature exploring the factors associated with adolescent engagement with CAMHS and the development of positive therapeutic alliance between teenagers and therapists. ${ }^{27-33}$ Using non-stigmatising language, facilitating the teenager's sense of being accepted and validated, and the therapist conveying respect were consistent themes that emerged in this literature.

User-led research has highlighted how the borderline personality disorder category is often perceived as invalidating. ${ }^{3,23-25}$ If this is the case, then revisiting Marsha Linehan's explanation of some of the features associated with the diagnosis (i.e. autonomic hypersensitivity + invalidating environment $=$ borderline personality disorder) ${ }^{34}$ leads to a conclusion with an unfortunate ironic twist - there is a clear risk of iatrogenically repeating the individual's experience of being invalidated by conceptualising their difficulties as a 'disorder'. Only if the diagnosis were shown to be uncontentiously valid and demonstrably clinically useful would, arguably, such a possible adverse outcome be a justifiable price to pay. Currently, a critical reading of the available literature regarding the conceptual and empirical validity, clinical utility and acceptability of the borderline personality disorder construct, particularly when applied to younger age groups, does not substantiate a comfortable or confident utilisation of the diagnosis in everyday CAMHS clinical practice. The majority of respondents who participated in the survey appear to share similar conclusions. And, as reflected in the findings of this survey, there appears to be a general scepticism about the added value of conceptualising the difficulties these teenagers are presenting with in borderline personality disorder diagnostic terms.

Our survey showed that consultant child and adolescent psychiatrists view borderline personality disorder category with, at best, scepticism when applied to young populations. Therefore, we recommend its use in children and adolescents be approached with caution.

\section{About the author}

Mark Griffiths is a consultant child and adolescent psychiatrist at Newport, Gwent, UK.

\section{References}

1 Aronson T. Historical perspectives on the borderline concept: a review and critique. Psychiatry 1985; 48: 209-22.

2 Bleiberg E. Borderline disorders in children and adolescents: the concept, the diagnoses and the controversies. Bull Menninger Clin 1994; 58: 169-96.

3 Wright K, Haigh K, McKeown M. Reclaiming the humanity in personality disorder. Int J Ment Health Nurs 2007; 16: 236-46.

4 Miller A, Muehlenkamp JJ, Jacobson C. Fact or fiction? Diagnosing borderline personality disorder in adolescents. Clin Psychol Rev 2008; 28: 969-81

5 Chanen A, Jovev M, Djaja D, McDougall E, Rawlings D. Screening for borderline personality disorder in outpatient youth. J Pers Disord 2008; 22: $353-64$

6 Shapiro T. Debate forum: resolved - borderline personality disorder exists in children under 12. J Am Acad Child Adolesc Psychiatry 1990; 29: 478-83.

7 American Psychiatric Association. Diagnostic and Statistical Manual of Mental Disorders, 4th edn (DSM-IV). American Psychiatric Association, 1994

8 World Health Organization. The ICD-10 Classification of Mental and Behavioural Disorders: Clinical Descriptions and Diagnostic Guidelines. World Health Organization, 1992

9 Wiggins O, Schwartz M. The limits of psychiatric knowledge and the problem of classification. In Philosophical Perspectives on Psychiatric Classification (ed J Sadler, O Wiggins, M Schwartz): 89-103. Johns Hopkins University Press, 1994.

10 Kupfer D, First M, Regier D. A Research Agenda for DSM-V. American Psychiatric Association, 2002.

11 Fulford B, Thornton T, Graham G. Oxford Textbook of Philosophy and Psychiatry. Oxford University Press, 2006.

12 Sadler J. Values and Psychiatric Diagnosis. Oxford University Press, 2005

13 Kendler KS. Toward a scientific psychiatric nosology: strengths and limitations. Arch Gen Psychiatry 1990; 47: 969-73.

14 Bolton D. What is Mental Disorder? An Essay in Philosophy, Science and Values. Oxford University Press, 2008.

15 Kendall R, Jablensky A. Distinguishing between the validity and utility of psychiatric diagnoses. Am J Psychiatry 2003; 160: 4-12. 
16 Bernstein D, Cohen P, Skodol A, Bezirganian S, Brook J. Childhood antecedents of adolescent personality disorders. Am J Psychiatry 1996; 153: 907-13.

17 Bondurant H, Greenfield B, Tse SM. Construct validity of the adolescent borderline personality disorder: a review. Can Child Adolesc Psychiatr Rev 2004; 13: 53-7.

18 Meijer M, Goedhart A, Treffers P. The persistence of borderline personality disorder in adolescence. J Pers Disord 1998; 12: 13-22.

19 Mattanah J, Becker D, Levy K, Edell W, McGlashan T. Diagnostic stability in adolescents followed up 2 years after hospitalization. Am J Psychiatry 1995; 152: 889-94

20 Garnet K, Levy K, Mattanah J, Edell W, McGlashen T. Borderline personality disorder in adolescents: ubiquitous or specific? Am Psychiatry 1994; 151: 1380-82.

21 Westen D, Shedler J, Durrett C, Glass S, Martens A. Personality disorder diagnoses in adolescence: DSM-IV axis II diagnoses and an empirically derived alternative. Am J Psychiatry 2003; 160: 952-66.

22 Bemporad J, Cicchetti D. Borderline syndromes in childhood: criteria for diagnosis. Am J Psychiatry 1982; 139: 596-601.

23 Hoffman P, Buteau E, Hooley J, Fruzetti A, Bruce M. Family members' knowledge about borderline personality disorder: correspondence with their levels of depression, burden, distress and expressed emotion. Fam Process 2003; 42: 469-78.

24 Castillo HA. Dangerous diagnosis: a user-led survey of people with a personality disorder diagnosis finds a group more sinned against than sinning. Mental Health Today 2003; 3 (February): 27-30.
25 Nehls N. Borderline personality disorder, gender stereotypes, stigma, and limited system of care. Issues Ment Health Nurs 1998; 19: 97-112.

26 Bateman A, Fonagy P. Psychotherapy for Borderline Personality Disorder: Mentalization-Based Treatment. Oxford University Press, 2004.

27 Garcia JA, Weisz JR. When youth mental health care stops: therapeutic relationship problems and other reasons for ending youth outpatient treatment. J Consult Clin Psychol 2002; 70: 439-43.

28 Dubow E, Lovko K, Kauasch D. Demographic differences in adolescents' health concerns and perceptions of helping agents. J Clin Child Psychol 1990: 19: 44-54.

29 Wilson C, Deane F, Biro V, Ciarrochi J. Youth Barriers to Help-Seeking and Referral from General Practitioners. Illawarra Division of General Practice and University of Wollongong, Illawarra Institute for Mental Health, 2003.

30 Rickwood D, Deane F, Wilson C, Ciarrochi J. Young people's help-seeking for mental health problems. Aust e-J Adv Ment Health 2005; 4: 1-34.

31 Thompson SJ, Bender K, Lantry J, Flynn PM. Treatment engagement: building therapeutic alliance in home-based treatment with adolescents and their families. Contemp Fam Ther 2007; 29: 39-55.

32 Everall R, Paulson B. The therapeutic alliance: adolescent perspectives. Counselling and Psychotherapy Research 2002; 2: 78-87.

33 Street C, Svanberg J. Where Next? New Directions in In-patient Mental Health Services for Young People. Young Minds, 2003.

34 Linehan M. Dialectical behavioural therapy: a cognitive behavioural approach to parasuicide. J Pers Disord 1987: 1: 328-33.

\title{
Personality disorder: challenges in service development in the light of the new NICE guidelines
}

\author{
Christopher Garrett, ${ }^{1}$ Tennyson Lee, ${ }^{2}$ Samantha Blackburn, ${ }^{2}$ Leanne Priestly, ${ }^{1}$ Kamaldeep Bhui ${ }^{3,4}$
}

The Psychiatrist (2011), 35, 22-26, doi: 10.1192/pb.bp.109.029249

\begin{abstract}
${ }^{1}$ East London NHS Foundation Trust; ${ }^{2}$ DeanCross Tower Hamlets

Personality Disorder Service, East London NHS Foundation Trust; ${ }^{3}$ Centre for Psychiatry, Barts and The London School of Medicine and Dentistry; ${ }^{4}$ Centre for Applied Research and Evaluation International Foundation (Careif), London
\end{abstract}

Correspondence to Tennyson Lee (tennyson.lee@eastlondon.nhs.uk)

\begin{abstract}
Aims and method Using the National Institute for Health and Clinical Excellence (NICE) guidelines on borderline personality disorder as a framework, we describe the profile of the first 100 individuals referred to a personality disorder service in London, captured through the use of record review and case study.

Results The referral population ethnic profile does not match the wider population of the borough; a third of the borough is Bangladeshi, but only $9 \%$ of those referred to the service are. Of those diagnosed with borderline personality disorder and on psychotropic medications, only one person had a clear current indication based on NICE guidelines. Of the 100 individuals who were referred to the service, a quarter were accepted to the programme; a third either did not want to proceed with the assessment or were unprepared for an intensive programme.

Clinical implications The under-representation of Black and minority ethnic individuals in referrals in the peresonality disorder service needs to be actively addressed. Interventions are required to support psychiatrists in reviewing their prescribing practice regarding individuals with borderline personality disorder. Access to the service needs to be improved.
\end{abstract}

Declaration of interest None.
The National Institute for Health and Clinical Excellence (NICE) guidance on borderline personality disorder has wide implications for psychiatric services. ${ }^{1}$ Key priorities outlined in this document include access to service, autonomy and choice, developing an optimistic and trusting relationship, and managing endings and transitions. General 